\section{Is the Masticatory Function Changed in Patients with Temporomandibular Disorder?}

Carolina Almeida Rodrigues, Melissa de Oliveira Melchior, Laís Valencise

Magri, Wilson Mestriner Jr., Marcelo Oliveira Mazzetto
Department of Restorative Dentistry, Ribeirão Preto School of Dentistry, USP - University of São Paulo, Ribeirão Preto, SP, Brazil

Correspondence: Carolina Almeida Rodrigues, Rua Benjamin Anderson Stauffer, 901, Jardim Botânico, 14021-617, Ribeirão Preto/SP, Brasil. Tel: +55-16-3315-4020. e-mail: carolinaalmeidarodrigues@ hotmail.com

\begin{abstract}
Patients with temporomandibular disorders (TMD) often complain and have limitation in masticatory function, which can be affected by a complex interaction of factors. The aim of this study was analyze the masticatory function in patients with TMD using surface electromyography (EMG) and masticatory efficiency (ME). Twenty-seven patients with TMD and 25 considered control (n), aged between 18 and 60 years, paired by age and gender, were evaluated according to RDC/TMD. In both groups were performed: EMG with chewing gum, clinical evaluation of habitual chewing with stuffed cookie (CE) (number of chewing strokes and time) and analysis of ME with fuchsin beads. Nonparametric statistical analyses were used (Mann-Whitney) for comparisons between groups, with $5 \%$ significance level. For all variables, the TMD group showed higher values than the control, with statistical significance for ME $(p<0.0001)$, number of chewing strokes $(p=0.04)$, chewing time ( $p=0.009)$, right masseter EMG activity $(p=0.05)$, left masseter $(p=0.005)$, right anterior temporal $(p=0.05)$ and left anterior temporal $(p=0.001)$. The conclusion is that patients with TMD showed changed chewing pattern, but without impairment of masticatory function.
\end{abstract}

Key Words: temporomandibular joint disorders, mastication, stomatognathic system, electromyography.

\section{Introduction}

The temporomandibular disorder (TMD) is characterized by a set of signs and symptoms: pain in temporomandibular joints, preauricular area and/or masticatory muscles, which worsen or are elicited during jaw function and palpation. Limitation or deviation in mandibular movements and joint noises may also be related to the clinical symptoms of TMD (1).

Patients with TMD often complain and have limitation in masticatory function, which can be affected by a complex interaction of direct (pain with dental origin, missing teeth, dentures) and indirect factors (disk displacements, mandibular hypermobility, individual susceptibility, etc.). Strength, movements and chewing time are influenced by the morphology, health status of orofacial structures and functional/adaptive capacity of the masticatory muscles and temporomandibular joints, apart from the characteristics of the food (2-4).

It is possible that the severity of TMD affects the adaptability and coordination of mandibular movements as a conscious or unconscious attempt to avoid noxious stimuli in proprioceptive scheme, reflecting on the chewing pattern. It has been suggested that the offsets made to perform the masticatory function in adverse conditions, even reflexively, are not always effective and may contribute to the progression of TMD in the long term. In the evaluation of chewing, the reasons that lead the individual to perform the functions in a certain form must be identified and allow the professional to plan specific therapies for each case. Therefore, objective methods have been employed to study it, such as surface electromyography (EMG), analysis of masticatory efficiency and myofunctional protocol ratings (5).

EMG has been considered an important method of analysis of the orofacial muscles because it is a device that involves the detection and records the electrical potential generated by the muscle fibers. It makes possible to know when and how a muscle is activated and also to define the coordination of different muscles involved in the movement. In patients with TMD occur changes in enrolment of the muscle as a compensatory mechanism for relieving pain or the asymmetrical muscle enrolment may precede the development of muscle pain symptoms $(6,7)$.

The masticatory efficiency (ME) can be defined as the ability to shred a given portion of food in a certain time and may be measured by the individual's ability to fragment natural or artificial foods. A colorimetric method was developed to evaluate ME by spectrophotometry, which measures the color intensity of the granules (beads/ pellets) encapsulated with fuchsin. This method is effective to evaluate ME because it is inexpensive, does not dissolve in saliva, does not vary, no loss during the evaluation, is biocompatible, accurate, fast, its physical properties are constant and is reliably reproduced (8-10). 
The aim of thisstudy was analyze the masticatory function in patients with TMD using surface electromyography (EMG) and masticatory efficiency (ME).

\section{Material and Methods}

\section{Study Design and Sample Description}

This was an observational cross-sectional study. The sample consisted of 27 patients with TMD, diagnosed according to the criteria of the RDC/TMD - Axis I, and 25 patients used as control paired by age and gender (11). This study was approved by the institutional Ethics Committee under protocol 08982312.6.0000.5419. Patients aged between 18 and 60 years old and without any treatment for TMD were included in the study. Patients wearing denture, toothless or major dental absences, patients with trauma history in head and neck, with psychiatric and/ or neurological disorders, with rheumatic diseases, using drugs (antidepressants, muscle relaxants, anxiolytics and anticonvulsants) and pregnant women were also excluded.

\section{Surface Electromyography}

After sample selection, the volunteers were submitted to EMG examination (Myotrace 400; Noraxon Inc., Scottsdale, AZ, USA, of masseter and temporalis muscles (anterior portion) bilaterally. EMG recording and analysis were performed according to the equipment protocol, using disposable 10-mm-diameter and 21-mm-lenght bipolar surface silver/silver chloride electrode ( $\mathrm{Hal}$ Ind. Com. Ltda., São Paulo, SP, Brazil). After cleaning the skin with $70 \%$ alcohol, the electrodes were positioned on the parallel bundles of muscle fibers and a disposable reference electrode was positioned approximately $10 \mathrm{~mm}$ above the glabella. The EMG examination was performed in a silent room with low lighting and the patient seated in a comfortable chair with his/her feet flat on the floor, the back against the chair backrest and gaze parallel to the ground ("the horizon"), consisting of three moments: habitual chewing for $40 \mathrm{~s}$, right unilateral chewing for 20 $\mathrm{s}$ and left unilateral chewing for $20 \mathrm{~s}$.

\section{Speech Therapy Assessment (Chewing)}

After EMG, masticatory function was clinically assessed by an experienced and calibrated speech therapist. The patients were instructed to chew in the habitual way a stuffed cookie (Nestlé, São Paulo, SP, Brazil), and the evaluation was recorded on video, with the same posture used for the electromyography exam. In this analysis the following aspects were considered: the bite, the grinding (chronic bilateral alternating, simultaneous bilateral, unilateral), the chewing time and the number of chewing strokes. The type and the chewing time were determined in the analysis of recorded videos where two assessors timed the chewing time and rated the mastication.

\section{Masticatory Efficiency Assessment}

This test was performed by the average of 2 chewing capsules containing fuchsin (beads), in habitual way for 20 s each. The patients were instructed to chew the beads after the speech therapy assessment with the cookie, aiming to remove the neuromuscular memory rest. The evaluation was performed in the same conditions and posture of the previous analyses, but without touching the head. These capsules comprise a special type of granule used as simulator for food testing. They comprised lactose (20.60 g), crystallized cellulose (36.85 g), corn starch (17:10 g), sucrose $(10.17 \mathrm{~g})$, hydrogenated oil $(8.05 \mathrm{~g})$, basic fuchsin $(0.16 \mathrm{~g})$, water $(60 \mathrm{~mL})$ and were coated with Eudragit E-100 with $5 \%$ acetone $50 \mathrm{~mL}$ (Rohm Pharma $\mathrm{GmbH}$, Weiterstadt, Germany). After chewing the capsule, its contents were sent to the laboratory and dissolved in $5 \mathrm{~mL}$ of water, by constant mixing for $30 \mathrm{~s}$. Then, the solution was filtered through qualitative filter paper and the extracted dye measured in nanometers $(\mathrm{nm})$ using a Beckman DU-7 UV-Visible Spectrophotometer (Beckman Inc., Palo Alto, CA, USA). The determination of ME was quantified by conversion to nanometer the extracted concentration of fuchsin (10).

\section{Statistical Analysis}

Data were compared between groups (control versus TMD) by non-parametric statistics (Mann-Whitney test) with a 5\% significance level (p).

\section{Results}

In the sample, both groups (TMD and control) showed higher prevalence of female gender (TMD: 22 women and 5 men; control: 18 women and 7 men) with a mean of age around 33.05 years old (TMD: $35.7 \pm 9.8$, control: $30.4 \pm 11.5$ ). Clinical assessments by RDC/TMD established the diagnostic classification of the sample as follows: 3 patients (11\%) were diagnosed with disc displacement (Group II), 2 (8\%) with degenerative changes (Group III), 12 (44\%) with myofascial pain associated with displacement of the disc (I and II), three (11\%) with displacement of the disc and degenerative changes (II and III), 2 (8\%) with myofascial pain and degenerative disorders (I and III ) and 5 (18\%) with the combination of the three conditions (I, II and III) (RDC/TMD).

In the ME assessment, the TMD group showed higher values $(0.38 \pm 0.3)$ compared to the control $(0.10 \pm 0.03)$, with a statistically significant difference $(p<0.0001)$. In relation to the total number of chewing strokes, higher values for the TMD group (63 \pm 16.7$)$ and control $(50 \pm 9.7)$ were found, with a statistically significant difference $(p=0.04)$. Similar result was obtained for the time (s) to chew the stuffed 
cookie, with higher values for the TMD group $(54.1 \pm 14.4)$ compared to the control $(45.4 \pm 8.2)$, with a statistically significant difference $(p=0.009)$ (Table 1$)$.

In EMG assessment during the habitual chewing, it was possible to observe that the control showed higher balance between the activities of the studied muscles, different from the TMD group, which showed greater activity of the anterior temporal muscles compared to masseter. All tested muscles showed greater activity in the TMD group compared to the control, with a statistically significant difference $(p=0.05)$ (Table 2).

\section{Discussion}

Important vital functions, such as chewing, swallowing and speech are directly related to the stomatognathic system. When the equilibrium of the system is changed and exceeds the patient's tolerance, a physiological collapse may occur due to inability of bearing excessive functional loads without the occurrence of discomfort, pain and/or compensation (12).

When patients with signs and symptoms of TMD were evaluated during food chewing with various consistencies (capsule system, Bono cookie and chewing gum), they showed significant differences when compared to the control. It was observed that these patients showed higher $M E$, more chewing strokes, increased chewing time (Table 1) and increased EMG activity of masseter and temporalis muscles (Table 2), and featured an altered chewing pattern.

It is known that in presence of pain the mandibular kinematics may suffer changes, affecting the neuromuscular

Table 1. Table 1. Median, standard deviation and p-value: masticatory efficiency $(\mathrm{mg} / \mathrm{mL})$, number of chewing strokes and chewing time (s). Mann-Whitney

\begin{tabular}{lccc}
\hline Day & TMD & Control & $p$ \\
\hline Masticatory efficiency $(\mu \mathrm{g} / \mathrm{mL})$ & $0.38( \pm 0.3)$ & $0.10( \pm 0.03)$ & $<0.0001^{*}$ \\
Number of chewing strokes & $63( \pm 16.7)$ & $50( \pm 9.7)$ & $0.04^{*}$ \\
Chewing time (seconds) & $54.1( \pm 14.4)$ & $45.3( \pm 8.2)$ & $0.009^{*}$ \\
\hline
\end{tabular}

*Statistically significant.

Table 2. Median, standard deviation and p-value of electromyographic activity of masseter and temporalis muscles during habitual mastication with chewing gum in comparison of TMD group versus control

\begin{tabular}{lccc}
\hline Muscle & TMD & Control & $p$ \\
\hline Right masseter & $17.3( \pm 7.4)$ & $15.9( \pm 14.9)$ & $0.05^{*}$ \\
Left masseter & $16.4( \pm 6.8)$ & $13.3( \pm 15.3)$ & $0.005^{*}$ \\
Right temporalis & $20.9( \pm 10.1)$ & $15.3( \pm 7.9)$ & $0.05^{*}$ \\
Left temporalis & $23.8( \pm 13)$ & $14.8( \pm 10)$ & $0.001^{*}$ \\
\hline
\end{tabular}

*Statistically significant. mechanism, which enroll differently the muscles and other structures associated with the stomatognathic system $(13,14)$. Patients with TMD may limit the mouth movements or perform them slowly when compared with healthy people (15). This limitation of motion aims to avoid the exacerbation of the pain, which could explain the higher chewing time and the higher number of chewing strokes in patients with TMD, because they usually chew more carefully (Table 1). Similar results were obtained during speech therapy assessments, which showed a higher difficulty and a lower ability to chew in TMD patients, besides a higher chewing time (2).

EMG is considered an important method for evaluating functionality of the masticatory muscles and is able of measuring the generated electrical potential by quantifying the electromyogram activity and allowing to verify whether the neuromuscular system is in equilibrium, besides assessing muscle coordination during dynamic activities (16). The literature shows that patients with moderatesevere TMD usually have increased EMG activity associated with lower precision on muscle coordination and increased function during lateral jaw movements, both working and balancing sides $(2,12)$. One study showed different results, in which lower EMG activity during chewing was found (13). The masticatory function involves complex movements of the stomatognathic system, and different muscle groups are activated at different times, which vary according to the food consistency and the presence/absence of pain. Such variations may explain the different results from the EMG activity in patients with TMD found in the scientific literature $(6,12,14)$.

Another interesting finding in this study was the higher activation of the anterior temporal muscle when compared with the masseter in patients with TMD (Table 2). Similar results were found in other studies, which related this increased activation with the presence of more effective occlusal contacts on anterior teeth $(2,6)$. The pain can also cause changes in the mandibular kinematics, which could alter the intermaxillary relationship and consequently the chewing pattern (13). Possibly these changes may be present in patients with TMD and could explain this higher activation of the temporal muscle.

It was observed that TMD patients had higher ME when compared with control (Table 1). This finding does not mean that in TMD the masticatory function is better, it is possible to suggest that in this condition is required a muscle compensation to perform this function, because of the pain and the limited movements, causing a higher force during the chewing (12). In addition, the bite force in TMD could not be decreased; some patients usually exhibit 
this increase when compared with healthy people; in both cases there is probably an imbalance in the structures of the stomatognathic system $(18,19)$.

Some authors found quite different results, they showed a decreased ME in patients with TMD when compared with control. This decreased ME could be related with occlusion and these authors suggested a strong association between the occlusal factor and ME. The dental absences and malocclusion affect directly the chewing pattern, which may cause a neuromuscular imbalance $(20,21)$. However, it is not possible to associate the occlusal factor with the presence of TMD and consequently the changed ME. In this study, it was found that patients with TMD have an increased $M E$, but the occlusal factor was not evaluated. There seems to be a relationship between the occlusion and $\mathrm{ME}$, though it must be considered that the mandibular kinematics is controlled by the action of the masticatory muscles, which may be compromised by the pain in TMD. A study showed that patients with disc displacement with or without reduction have a decreased masticatory function (22).

A study showed that the chewing performance of subjects with disk displacement was lower than in healthy $\approx$ patients, but in case of severe pain in the orofacial region, the performance was similar to that of healthy patients. The authors show that in presence of pain, there is a tendency to chew the food into smaller pieces and more cautiously, resulting in a higher masticatory time and consequently a greater masticatory efficiency (23).

Given the set of changes that involve the masticatory function of the neuromuscular and TMD patients, the same analyzes were conducted with different consistency materials during the evaluation of patients with TMD and control. There seems to be a clear relationship with this changed masticatory pattern. Greater neuromuscular enrolment is required to perform the functions and chewing requires larger efforts that could lead to an increased ME compared with healthy patients $(2-4,14)$.

However, orofacial behaviors are often determined by sensory experience, and the pain is a strong factor for adapting these behaviors in order to maintain the function and system protection. But these adjustments or compensations may have consequences in time, accentuating the pain and causing disabling and even irreversible injuries, according to some authors $(5,24)$. Thus, the importance of assessing not only the pain but also the entire myofunctional mechanism involved and its consequences is undeniable to properly guide the therapeutic management, for the purpose of treatment and above all to avoid progression of signs and symptoms (16).

It is important to point out that even if ME and EMG activity are not reduced in TMD condition, it does not mean absence of pain and/or difficulty during the masticatory function, which exerts great demand on the stomatognathic system. According to the evaluated parameters, it is possible to conclude that patients with TMD have an altered chewing pattern when compared to healthy individuals, without compromising the masticatory function, due to a greater enrolment of neuromuscular and adaptive changes to preserve the vital function.

\section{Resumo}

Pacientes com disfunção temporomandibular (DTM) frequentemente se queixam e apresentam limitação na função mastigatória, a qual pode ser afetada por uma complexa interação de fatores. 0 objetivo deste estudo foi analisar a função mastigatória de pacientes com DTM em comparação a sujeitos controle por meio das técnicas de eletromiografia de superficie e eficiência mastigatória (EM). 27 pacientes com DTM e 25 considerados controle (n), com idade entre 18 e 60 anos, pareados por idade e gênero, foram avaliados segundo o RDC/TMD. Em ambos os grupos foram realizados: exame de eletromiografia de superfície da mastigação habitual com goma de mascar (EMG), avaliação fonoaudiológica da mastigação habitual com biscoito recheado (AF) (número total de golpes mastigatórios e tempo) e análise da eficiência mastigatória com o sistema de cápsulas compostas por beads de fucsina (EM). Foi empregada estatística não paramétrica (Mann-Whitney) para a comparação intergrupos, adotando-se $5 \%$ como nivel de significância. Para todas as variáveis estudadas o grupo DTM apresentou valores superiores em relação ao grupo controle, com significância estatística: $\mathrm{EM}(\mathrm{p}<0,0001)$, número total de golpes $(\mathrm{p}=0,04)$, tempo de mastigação $(p=0,009)$, atividade eletromiográfica masseter direito $(p=0,05)$, masseter esquerdo $(p=0,005)$, temporal anterior direito $(0,05)$ e temporal anterior esquerdo $(p=0,001)$. Conclui-se que pacientes com DTM apresentam um padrão mastigatório alterado porém, sem comprometimento da função mastigatória.

\section{References}

1. Scrivani SJ, Keith DA, Kaban LB. Temporomandibular disorders. N Engl J Med 2008;359:2693-2705.

2. Ferreira $C L$, Machado $B C$, Borges $C G$, Rodrigues da Silva $M A$, Sforza C, De Felício CM. Impaired orofacial motor functions on chronic temporomandibular disorders. J Electromyogr Kinesiol 2014;24:565571.

3. Hatch JP, Shinkai RS, Sakai S, Rugh JD, Paunovich ED. Determinants of masticatory performance in dentate adults. Arch Oral Biol 2001;46:641-648.

4. Mioche L, Bourdiol P, Monier S. Chewing behavior and bolus formation during mastication of meat with different textures. Arch Oral Biol 2003;48:193-200.

5. Ferreira CLP, Rodrigues da Silva MAM, De Felício CM. Orofacial myofunctional disorder in subjects with temporomandibular disorder. Cranio 2009;27:268-274.

6. Tartaglia GM, Lodetti G, Paiva F, De Felicio CM, Sforza C. Surface electromyography assessment of patients with long lasting temporomandibular joint disorder pain. J Electromyogr Kinesiol 2011;21:659-664.

7. Nielsen IL, McNeill C, Danzig W, Goldman S, Levy J, Miller AJ. Adaptation of craniofacial muscles in subjects with craniomandibular disorders. Am J Orthod Dentofacial Orthop 2009;97:20-34.

8. De Abreu RA, Pereira MD, Furtado F, Prado GP, Mestriner Jr. W, Ferreira LM. Masticatory efficiency and bite force in individuals with normal occlusion. Arch Oral Biol 2014;59:1065-1074.

9. Pocztaruk RL, Frasca LC, Rivaldo EG, Fernandes EL, Gavião MB. Protocol for production of a chewable material for masticatory function tests (Optocal-Brazilian version). Braz Oral Res 2008;22:305-310.

10. Escudeiro-Santos C, Freitas 0, Spadaro ACC, Mestriner Jr. W. Development of a colorimetric system for evaluation of the 
masticatory efficiency. Braz Dent J 2006:17:95-99.

11. Dworkin SF, LeResche L. Research diagnostic criteria for temporomandibular disorders: review, criteria, examinations and specifications, critique. J Craniomandibular Disord 1992;6:301-355.

12. De Felício CM, Ferreira CLP, Medeiros APM, Silva MAMR, Tartaglia GM, Sforza C. Electromyographic indices, orofacial myofunctional status and temporomandibular disorders severity: A correlation study. J Electromyogr Kinesiol 2012;22:266-272.

13. De Felicio CM, Mapello A, Sidequersky FV, Tartaglia GM, Sforza C. Mandibular kinematics and masticatory muscles EMG in patients with short lasting TMD of mild-moderate severity. J Electromyogr Kinesiol 2013;23:627-633.

14. Lobbezoo F, Van Selms MKA, Naeije M. Masticatory muscle pain and disordered jaw motor behavior: Literature review over the past decade. Arch Oral Biol 2006;51:713-720.

15. Bakke M, Hansdottir R. Mandibular function in patients with temporomandibular joint pain: a 3-year follow-up. Oral Surg Oral Med Oral Pathol Oral Radiol Endod 2008;106:227-234.

16. Mazzetto MO, Rodrigues CA, Magri LV, Melchior MO, Paiva G. Severity of TMD related to age, sex and electromyographic analysis. Braz Dent J 2014;25:54-58.

17. Tartaglia GM, Testori T, Pallavera A, Marelli B, Sforza C. Electromyographic analysis of masticatory and neck muscles in subjects with natural dentition, teeth-supported and implant-supported prostheses. Clin Oral Implants Res 2008;39:858-877.

18. Gomes SGF, Custodio W, Jufer JSM, Cury AADB, Garcia RCR. Correlation of mastication and masticatory movements and effect of chewing side preference. Braz Dent J 2010;21:351-355.

19. Pizzolato RA, Gavião MD, Berretin-Feliz G, Sampaio AC, Trindade Jr AS. Maximal bite force in young adults with temporomandibular disorders and bruxism. Braz Oral Res 2007;21:278-283.

20. Kobayashi T, Honma K, Nakajima T, Hanada K. Masticatory function in patients with mandibular prognathism before and after orthognathic surgery. J Oral Maxillofac Surg 1993;51:997-1001.

21. Kümbüloglu Ö, Saracoglu A, Bingöl P, Hatipoglu A, Özcan M. Clinical study on the comparison of masticatory efficiency and jaw movement before and after temporomandibular disorder treatment. Cranio 2013;31:190-201.

22. Sato $S$, Ohta $M$, Sawatari $M$, Kawamura $H$, Motegi K. Occlusal contact area, occlusal pressure, bite force, and masticatory efficiency in patients with anterior disc displacement of the temporomandibular joint. J Oral Rehabil 1999;26:906-911.

23. Peroz I, Tai S. Masticatory performance in patients with anterior disk displacement without reduction in comparison with symptom-free volunteers. Eur J Oral Sci 2002;110:341-344.

24. Peck CC, Murray GM, Gerzina TM. How does pain affect jaw muscle activity? The integrated pain adaptation model. Aust Dent J 2008;53:201-207.
Received August 18, 2014 Accepted December 17, 2014 